\title{
Category Classes: Flexible Classification and Evolution in Object-Oriented Databases
}

\author{
Erik Odberg* \\ Department of Computer Science \\ Norwegian Institute of Technology
}

\begin{abstract}
Object-oriented databases (OODBs) are believed to more naturally reflect the behavior and organization of real world objects. However, OODBs are mostly concerned about only the static aspects of object modeling. While real world objects typically may be multi-perspectived and evolve over time by changing classification and behavior, contemporary OODB models typically regard objects as instances of classes in such a way that classification (and thus behavior) is fixed at the time of creation.

This paper introduces the notion of an object role to denote a particular perspective of an object, corresponding to a class for which it is an instance. Roles may be dynamically added and removed from objects to reflect the way real world objects classify and evolve over time, and simultaneously change behavior. A category class is a special class which is associated with a predicate, and in this way describe constraints on how objects may evolve, as well as how objects may automatically gain and discard roles based on various criteria.
\end{abstract}

\section{Introduction and Motivation}

Object-oriented data models (and consequently object-oriented databases (OODBs)) are believed to better and more naturally reflecting the behavior and organization of real world phenomena, incorporating more of the real world semantics. Objects separate externally visible behavior from internal representation and implementation. Classes abstract over commonalities between objects, defining classifications of objects. Class hierarchies impose an organization of classes describing a conceptual specialization (i.e. that instances of one class may also be regarded as instances of more general superclasses) and inheritance of properties (i.e. that instances of one class will also contain properties as defined for superclasses) ${ }^{1}$.

However, most object models are only concerned about the static aspects of modeling, and not how objects may evolve over time. Objects are created as an instance of one class, which also serves to classify the object according to the predefined taxonomy, and to define its properties. Once created, objects cannot change classification or property possession. Naturally, this does not refiect very well the way the "real world" behaves: Real-world objects ("phenomena") may typically have different appearance (exhibited behavior) in different contexts of expectation, and may be perceived to classify differently (dependent on expectation). Phenomena are often highly dynamic, and evolve over time by changing classification and appearance.

The remaining part of this paper describes an extension to the traditional notion of object-orientation which naturally reflects the behavior of the "real world". Section 2 introduces the notion of an object role as a particular perspective of an object, and discusses how roles may be added to and removed from objects to allow objects to evolve. Section 3 defines a category class as a specialization of the

\footnotetext{
'Detailed address: Department of Computer Science, Norwegian Institute of Technology (NTH), N-7034 TrondheimNTH, Norway. Phone: +4773 594484. Fax: +47 73 594466. Email: eriko@idtunit.no

${ }^{1}$ The conceptual organization and property reuse dimensions of subclassing reflect the Scandinavian and American school of $O O$ thinking, respectively.
} 
class construct which may define restrictions on or enable certain evolutions of objects. Section 4 compares the approach with related work, while Section 5 concludes the paper and presents directions for further work.

\section{Objects with Roles}

The fundamental object model is "traditional" in that it adopts $\mathrm{C}++$ as the primary source of influence. Objects are created as instances of classes, which have an associated collection of properties. Properties may be attributes or methods, defined in a way which is similar to $\mathrm{C}_{++}$, and have a visibility which is either public (accessible by external clients of the object) or private (only accessible to method implementations for the class). The visibility is specified by keywords public and private, with public being default (in contrast to $\mathrm{C}++$ ). A notion of explicit relationships is supported to provide symmetric associations (with cardinalities) between two classes, and with special functionality for navigating over these. A class may have one or more superclasses (defining the class as a subclass of these). The subclass inherits the properties of the superclasses (retaining the visibility mode ${ }^{2}$ ), and may define additional properties or redefine inherited ones. The subclass also defines a specialization relationship with its superclasses, and so that instances of one class are also instances of all superclasses (transitively). Note that a class Class is the implicit superclass of all classes for which no superclass is defined; in this way a schema will consist of one connected class hierarchy.

\subsection{Object manipulation}

Persistent objects are manipulated (created, modified, deleted) through a corresponding C++ placeholder object. Placeholder objects are instances of $\mathrm{C}++$ persistent classes, which are generated from the schema specification. Each persistent $\mathrm{C}++$ class directly corresponds to a schema class, having the same name and properties, and providing additional functionality for persistent manipulation. Placeholders are acquired from the database by navigation over database relationships, by iteration over a set of persistent objects (possibly acquired through an associative query), or as a result from a method invocation.

Objects created as instances of persistent classes will not automatically persist in the database. This must be explicitly requested by invocation of a function MakePersist, associated with each persistent class. In this way instances of persistent classes may be both persistent and non-persistent, but may smoothly interact within the same application process. Object modifications are also "committed" persistently by invocation of MakePersist.

\subsection{Object roles}

Each class an object may be regarded an instance of will be denoted a role of this object. Each object role reflects a particular perspective of the object, a context of behavior which may be referenced by clients. More important, roles (i.e. class memberships) reflect characteristics of objects which may be independently gained and lost: Objects may evolve over time by changing role possession (i.e. the classification and associated properties) dynamically. Some roles may reflect specializations of other roles (corresponding to superclasses), however an important characteristic of the model is the ability for objects to possess roles corresponding to sibling classes. Between two sibling classes there is neither a subclassing relationship, nor is there a common subclass of these classes. We say that objects may contain multiple most-specific roles, or that objects are multi-perspectived. This is in contrast with traditional object-oriented models where each object is an instance of one class

\footnotetext{
${ }^{2}$ This is the same as public inheritance in $\mathrm{C}++$.
} 
which is decided upon creation, and all "roles" correspond to the creation class and its superclasses. There is no ability to have object evolve or assume additional most-specific roles.

The creation of an object will allocate a collection of roles (for all classes the object is an instance), and simultaneously assign a unique object identifier (OID) to the new object. New roles may be added, according to the class hierarchy, and in this way "extending" the object downwards. No new OID is assigned upon role addition, it is still the same object. Any role(s) may also be removed from the object (without deleting the object or affecting the OID), and which will also have the impact that all roles corresponding to subclasses of the denoted role class are removed as well. The strong notion of identity means that no object set may contain multiple occurrences of the same object, even if different roles are reganded. Furthermore, two operators for checking the identity of object references are provided: Two placeholders are object identical if they correspond to the same (persistent) object, while placeholders are object-role identical if they correspond to the same object and role.

Figure 1 illustrates an example class hierarchy.

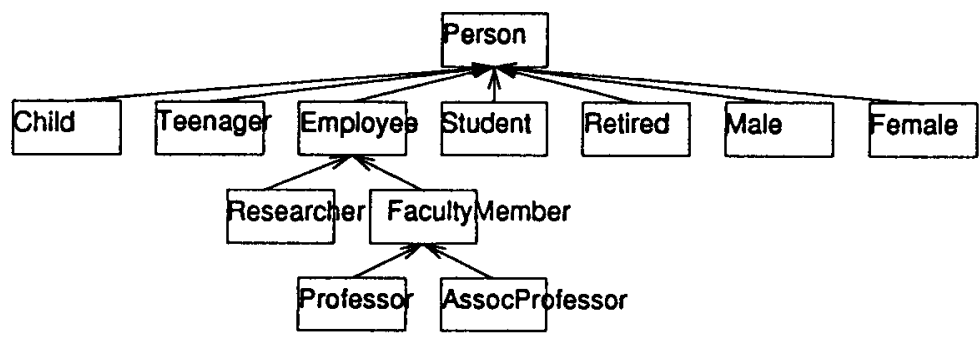

Figure 1: Class hierarchy

A person's "life" is described by the way roles are gained and lost, possibly possessing many different (most-specific) roles simultaneously. Some roles may be automatically gained and lost (for instance Child and Teenager due to age), while some must be explicitly added (as for instance Student and Employee). Some roles are inherently incompatible, and may not be possessed at the same time (for instance Female and Male, or Retired and Employee). Other roles may be possessed simultaneously, as for instance a female teaching assistant which is an instance of Female, Employee and Student, and behaves in different ways in these three roles.

\subsection{Object role manipulation}

The introduction of the role notion have implications to the functionality provided for object manipulation.

\subsubsection{Adding roles}

New roles are added to persistent objects through the add operator, which is applicable to all placeholders:

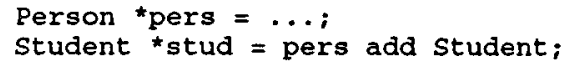


A role is always added as a specialization of some existing role, however may perfectly well be added as another "branch" of the object, and so that the object does not have to be an instance of a single most-specific class. For instance, the following example illustrates a person which is simultaneously also a student and an employee:

$$
\text { Employee *emp = stud add Employee; }
$$

It must be noted that there is no constraint on what is the class of the object which is the basis for the role addition: In principle any role may be added to any object, however Section 3 will show how restrictions may be imposed.

The add operator returns a pointer to a $\mathrm{C}++$ placeholder object as an instance of the class corresponding to the added role. This means that the placeholders referenced by pers, stud and emp, which correspond to the same persistent object, need not be the same $\mathrm{C}++$ objects $^{3}$. Rather, they reference different perspectives of the same (persistent) object. Application programs must ensure, in order to avoid mutation conflicts, that objects are mutated through only one perspective (placeholder) at the time. That is, applications should preferably synchronize with the database (by invoking MakePersist) before the new role is added. The DBMS has no other means to ensure that multiple placeholders within the same process are maintained consistently wrt. mutations. Note that a role addition is not reflected persistently unless MakePersist is explicitly invoked.

If a role is added to an object which already does possess this role, the object is not affected by the addition. This means that an object may have only one role corresponding to each class. If a role is possessed by a persistent object, but not reflected by the actual placeholder, a new placeholder must be retumed. This is because the result of the add operation is expected to be an instance of the added class. Consequently, for the following operation no new role is added, but another placeholder (which may be the same as for stud) is retumed:

$$
\text { student *stud2 = emp add student; }
$$

It must be noted that some other approaches, notably Aspects [RS91] and Clovers [SZ89], allows for instantiating multiple roles of the same class. It is our position that this is unnatural modeling, and better refiected by the use of relationships between objects. Furthermore, there is an important problem wrt. identification of roles which reflect the same class.

A notion of a role constructor is provided for the initialization of new roles. Role constructors operate as ordinary constructors, but initialize only attributes directly associated (i.e. non-inherited) with the class of the role to be added. Zero or more role constructors may be associated with each class. If there is no visible role constructor defined for a particular class, this means that the class may not be added as a role to any existing object ${ }^{4}$ :

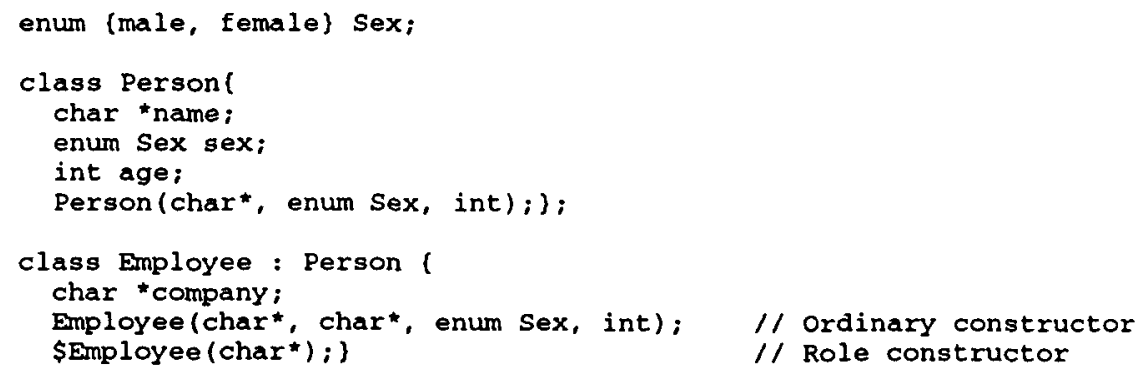

Similar to for object creation, role addition will thus take arguments as indicated by the role constructor:

Person ${ }^{\star}$ pers $=$ new person ("Donald Duck"', male, 60);
Employee "emp $=$ pers add employee ("Walt Disney Corp."');

${ }^{3}$ In fact, in this case they cannot be, as it is not possible to have one C++ object be an instance of both Student and Employe日, as there is no common subclass for these classes.

"As for ordinary $\mathrm{C}++$ constructors, if no role constructor is explicitly specified, a non-argument role constructor will by default be available. 
A role is always added as a specialization of some existing role, however may perfectly well be added as another "branch" of the object, and so that the object does not have to be an instance of a single most-specific class. For instance, the following example illustrates a person which is simultaneously also a student and an employee:

$$
\text { Employee *emp = stud add Employee; }
$$

It must be noted that there is no constraint on what is the class of the object which is the basis for the role addition: In principle any role may be added to any object, however Section 3 will show how restrictions may be imposed.

The add operator retums a pointer to a $\mathrm{C}++$ placeholder object as an instance of the class corresponding to the added role. This means that the placeholders referenced by pers, stud and emp, which correspond to the same persistent object, need not be the same C++ objects ${ }^{3}$. Rather, they reference different perspectives of the same (persistent) object. Application programs must ensure, in order to avoid mutation conflicts, that objects are mutated through only one perspective (placeholder) at the time. That is, applications should preferably synchronize with the database (by invoking MakePersist) before the new role is added. The DBMS has no other means to ensure that multiple placeholders within the same process are maintained consistently wrt. mutations. Note that a role addition is not reflected persistently unless MakePersist is explicitly invoked.

If a role is added to an object which already does possess this role, the object is not affected by the addition. This means that an object may have only one role corresponding to each class. If a role is possessed by a persistent object, but not reflected by the actual placeholder, a new placeholder must be retumed. This is because the result of the add operation is expected to be an instance of the added class. Consequently, for the following operation no new role is added, but another placeholder (which may be the same as for stud) is retumed:

$$
\text { student *stud2 = emp add student; }
$$

It must be noted that some other approaches, notably Aspects [RS91] and Clovers [SZ89], allows for instantiating multiple roles of the same class. It is our position that this is unnatural modeling, and better reflected by the use of relationships between objects. Furthermore, there is an important problem wrt. identification of roles which reflect the same class.

A notion of a role constructor is provided for the initialization of new roles. Role constructors operate as ordinary constructors, but initialize only attributes directly associated (i.e. non-inherited) with the class of the role to be added. Zero or more role constructors may be associated with each class. If there is no visible role constructor defined for a particular class, this means that the class may not be added as a role to any existing object ${ }^{4}$ :

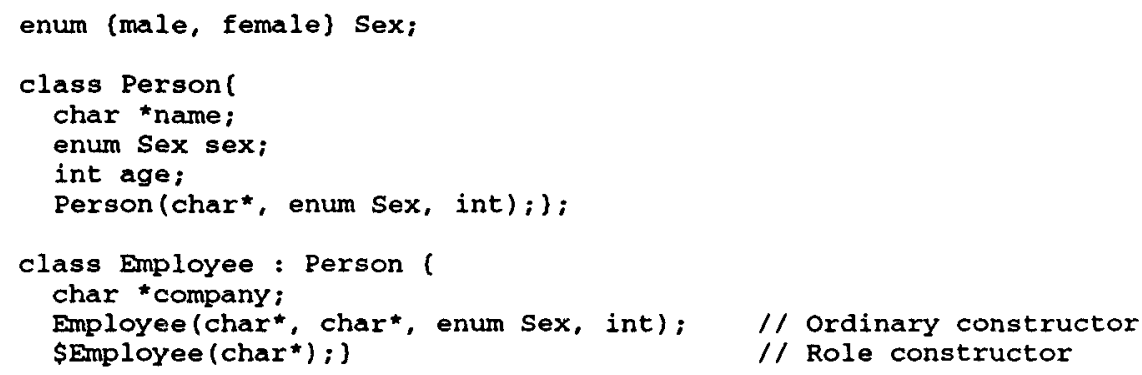

Similar to for object creation, role addition will thus take arguments as indicated by the role constructor:

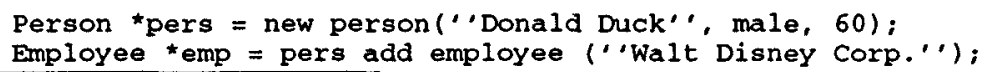

\footnotetext{
${ }^{3}$ In fact, in this case they cannot be, as it is not possible to have one $\mathrm{C}++$ object be an instance of both Student and Employee, as there is no common subclass for these classes.

As for ordinary $\mathrm{C}_{++}$constructors, if no role constructor is explicitly specified, a non-argument role constructor will by default be available.
} 


\subsubsection{Removing roles}

Roles may also be removed from objects, using the rem operator:

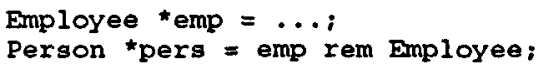

The rem operator removes the role corresponding to the indicated class from the object (provided it is contained), as well as all contained roles corresponding to subclasses. A pointer to the same object as an instance of the immediate superclass of the removed class is retumed ${ }^{5}$. In general, this means that another placeholder (another $\mathrm{C}++$ pointer) will be retumed. MakePersist must be invoked to have the role removal to be persistently reflected. Note that it is possible to remove a role from an object even if the placeholder is not an instance of the corresponding persistent class, as long as the role is possessed persistently:

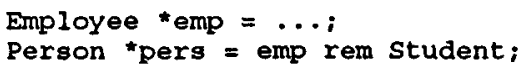

A notion of a role destructor is provided, similarly to ordinary destructors, to "clean up" data structures when some role is removed from an object. More interestingly, if there is no public role destructor associated with a class, the corresponding role may not be removed from the object by any application program.

\subsubsection{Reference coercion}

Object references may be coerced to another class, in the same way as in $\mathrm{C}++$. In this way the same (persistent) object may be inspected through another role and thus exhibiting itself differently. Coercions may be performed upwards (to a superclass, and thus restrict the set of available properties and/or change bindings for properties overridden by subclasses), downwards (to a subclass, and thus assuming additional properties), or to a sibling class (a class which is neither a superclass or subclass, and thus may provide a completely different set of properties). The following are some natural coercions which may originate using the class hierarchy of Figure 1:

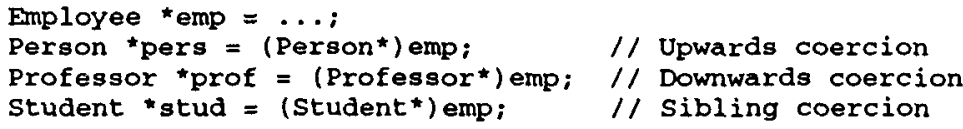

The coercion will retum a pointer to a $\mathrm{C}++$ placeholder object which is generally different from the original, as they reflect the same object as an instance of different persistent classes. Note that if the object does not have the role corresponding to the coercion, an exception will be raised (and the retum pointer is invalid). Naturally, this may only be checked at runtime.

\subsubsection{Placeholder construction}

When a placeholder is constructed to reflect a persistent counterpart, it will normally be created as an instance of the persistent class corresponding to the most-specific class of the persistent object (rather than the class of the reference). However, according to the model of roles an object may have multiple most-specific classes. This cannot be expressed within $\mathrm{C}++$, and thus the placeholder objects (reflecting references to persistent objects) must be created as instances of a unique persistent class. Different possibilities exist to decide about this class: It may the same as the class of the reference, but this will not work correctly in the presence of virtual functions. Rather, an object should be retumed as an instance of some most-specific class for which the persistent object is an

\footnotetext{
${ }^{5}$ If the removed class have muliple immediate superclasses, the rem operator will return a pointer to an instance of the class which is the most-specific (unique) common superclass of these classes.
} 
instance. This most-specific class may be selected arbitrarily by the system, or there may be some priority based on for instance which role was first (or last) added. Another possibility is to have application programs denote the class of the placeholder object by explicit coercion.

Virtual method invocations are bound to implementation dynamically (late binding), on the basis of the class of the object rather than the reference to the object. As there need not be a unique mostspecific class of the (persistent) object, virtual binding will be based on the placeholder object, in this way taking advantage of $\mathrm{C}++$ nutime provisions. This means that virtual binding is dependent on the strategy for placeholder object construction. Indeed, virtual binding is an inherent problem of the model, given the traditional interpretation. A more "natural" solution, may be to have virtual method implementations take into consideration which subclasses the (persistent) object is an instance of, and perform a computation based on this knowledge. In this way special "combination methods" may be implemented in terms of methods of multiple most-specific classes, or possibly explicitly selecting between these.

\section{Category Classes}

A category class is a specialization of the class construct with special abilities to restrict or enable object membership in the class through the association with a predicate. For ordinary classes, there are no restrictions on object creation and deletion, or role addition and removal, apart from the possible inhibition described by invisible (role) constructors or destructors. Fundamentally, any role may be added (explicitly) to any object, and any role possessed may be removed. Membership in category classes, however, depends on satisfaction of the category class predicate. For a manual category class, the explicit addition of the corresponding role to some object will only succeed if the predicate is satisfied at the time of addition. An automatic category class is different in that satisfaction of the predicate will automatically make the role be added to the object, however it will be lost if the predicate is later dis-satisfied.

In most respects category classes are similar to ordinary classes. They are organized in the same class hierarchy, and thus define inheritance and substitutability relationships with superclasses. Category classes associate with properties as ordinary classes, and object variables may reference instances of category classes (through the placeholder) just like instances of other classes. Category classes and ordinary classes are different variations of the same abstraction mechanism, with an additional ability for category classes to restrict or enable membership in powerful ways ${ }^{6}$. Category classes express special knowledge about phenomena evolution and classification in the domain of discourse, making the schema able to include more aspects of real-world semantics.

\subsection{Category class definition}

The general syntax of a category class definition goes as follows:

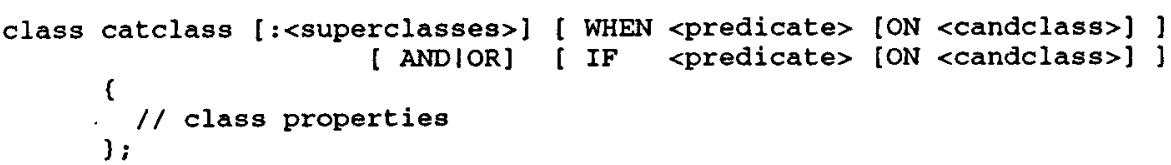

A category class (catclass) may have one or more superclasses, which may be ordinary or category classes. Manual category classes are defined by an IF expression, for which <predicate > describes a restriction to be satisfied to permit the explicit addition (using the add operator) of the corresponding role to some object. Automatic category classes are defined by a WHEN expression, for which <predicate > defines the criterion for the corresponding role automatically to be added to some object. IF and WHEN expressions may also be combined (using logical operators AND or OR), as will be explained below.

\footnotetext{
${ }^{6}$ An ordinary class may thus be regarded as a manual category class with an empty (i.e. True) predicate.
} 


\section{Candidate classes}

ON expressions define the candidate class of the particular category class. Only objects which possess the corresponding role (is an instance of the candidate class) are valid candidates for membership in the category class. Furthermore, category class predicates may only reference properties defined for the candidate class (or superclasses of this). The specification of a candidate class is optional; if no candidate class is defined, the immediate superclass of the category class will apply by default. If the category class has multiple superclasses, the lowest common superclass will be the effective candidate class. Most often, the candidate class is a superclass of the category class, and so that membership in the category class will reflect a specialization of the candidate class role. A candidate class may also be a sibling class of the category class, and so that a corresponding role addition may add an additional most-specific mole of the object. A candidate class which is a subclass of the category class is meaningless.

\section{Predicates}

Category class predicates may reference two aspects of objects; the properties defined for the candidate class, and the role possession of the object. Both public and private attributes may be referenced as part of the predicate specification. While clients of objects may only access the public part of class definitions, category class predicates are regarded a part of the definition of the class and thus may reference internal representation as well. Method-based predicates are permitted, but must be used with care as method invocations may have undesirable side-effects (Cfr. for instance [Odb92]). Moreover, automatic category class predicates are (at least conceptually) continuously evaluated for all candidate objects, which means that side-affect methods may have arbitrary affects on objects.

Property-based predicates are specified using ordinary C++ comparison operators and boolean operators \&\& (and), \| (or) and | (not), referencing the state of objects as well as constant values. Predicates are evaluated in the context of objects which are instances of the candidate class, with predicate attributes bound to the particular object. Role-based predicates specify requirements on the object possession of roles. Role possession is denoted through the name of the class, and using $\mathrm{C}++$ boolean operators any predicate over role possession may be defined to constrain (for manual) or enable (for automatic) membership in the category class. Attribute- and role-based expressions may also be combined in the same predicate. Examples of the different kinds of predicates are shown below.

\subsection{Manual category classes}

Manual category classes are defined through an IF expression, giving a predicate which must satisfied to permit the explicit addition of the corresponding role to an object (which must also possess the role corresponding to the candidate class). Furthermore, for all superclasses of the category class, the object must either already possess the corresponding role (be a member), or addition must be permitted (i.e. predicates possibly associated must be satisfied). This means that multiple roles may be added in one go. If for some reason role addition is not permitted, the operation fails and an error code is set. If addition succeeds, the role is contained until the object is deleted, the role is explicitly removed, or some automatic superclass predicate is no longer satisfied (Cfr. below). However, there is no requirement that manual category class predicates are satisfied after a successful addition.

Manual category classes are most commonly used to define constraints on object evolution, and thus implicitly define valid patterns of migration. Addition of a particular role to some object requires that it fulfills certain characteristics in terms of state and/or that it does/does not already possess a certain combination of roles. Manual category classes generally associate with additional properties, so that new attributes are typically initialized by a role constructor. 
For instance no person may become a student if not old enough:

class student : Person IF age $>=17$ oN Person (

...);

This predicate is attribute-based, but manual category class predicates may also be based on the possession of roles. For instance, all full or associate professors may be a $P h D$ promoter (however does not have to), while other faculty members may not:

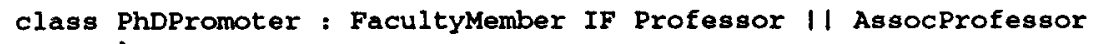

Note that PhDPromoter is neither a subclass nor superclass of Professor or AssocProfessor.

Manual category classes may also be defined on the basis of predicates referencing both attributes and role possession. For instance, to qualify for professorship you must have eamed a PhD degree and have more than 25 publications, or have been employed at the university (as a faculty member) more than 30 years:

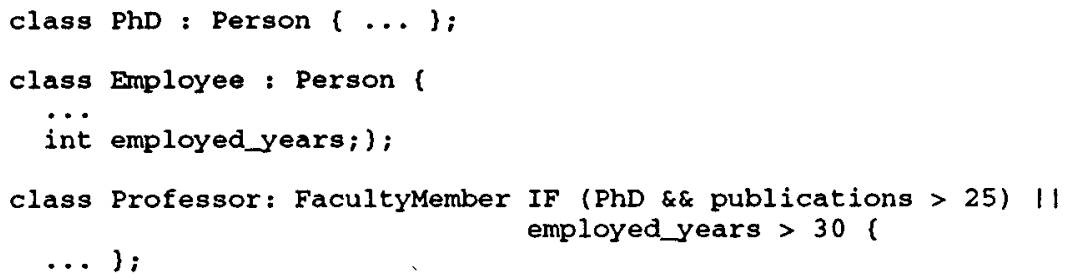

Note that, as Professor is a manual category class, promotion is not guaranteed even if the predicate is satisfied ${ }^{7}$.

\subsection{Automatic category classes}

Automatic category classes are defined by WHEN expressions, which define a criterion for objects to automatically assume the role corresponding to the category class. Logically, all objects which possess the candidate class role (i.e. is an instance of the candidate class) are (at least conceptually) continuously evaluated for possible predicate satisfaction. If the predicate is satisfied, and roles corresponding to superclasses either are possessed, or addition is permitted, the category class role (and possibly "missing" superclass roles) are assumed by the object. If at some time the predicate for the class, or for some automatic category superclass, is no longer satisfied the role will become invisible. The same applies if the candidate class role is lost. However, if the role possession is later re-enabled the same role (with the same state) will reappear, the role is not persistently removed. The same also applies for any subclasses of the automatic category class as well: Upon dis-satisfaction of an automatic category superclass predicate subclass roles will become invisible (superclass membership is a prerequisite for membership in subclasses), but will reappear upon predicate re-satisfaction. Note that, in contrast, for manual category classes predicates are only evaluated upon role addition, and upon role removal all roles (including for subclasses) are persistently removed.

In some cases, an object may satisfy the predicate of multiple automatic category classes, although membership in these may be mutually exclusive. In this case predicates will be conficting, and membership in either may prohibit membership in others. The system will make an arbitrary choice of class membership in these cases. Note that the system is not able to detect that such a situation may possibly arise, and thus no waming may be given at schema definition time.

While any (category) class may associate with both methods and attributes, automatic category classes often tend not to add attributes in addition to those inherited from superclasses. The reason is that automatic category class roles are implicitly added, and thus only non-argument role constructors may

\footnotetext{
${ }^{7}$ Which means promotion should probably not be expected for just waiting passively for 30 years ...
} 
be invoked for the initialization of the role. More commonly, however, automatic category classes define new methods, or redefine inherited ones. In this way special behavior may be associated with objects as a member of automatic category classes, and for which the association is dependent on predicate satisfaction. Automatic category classes are mostly concemed about the membership (extent) dimension and for which flexible specification is possible. This also reflects the typical use of automatic category classes, described below.

\subsubsection{Using automatic category classes}

Automatic category classes may be used in different ways. Typically they are used to describe one or more partitions of a superclass based on values for attributes of the superclass, and so that each partition class contain a subset of the objects in the superclass. Partition classes may be disjoint or overlapping, and may be complete or non-complete partitions. Specification of completeness and disjointness is left to the schema designer, and is dependent on the ability to define predicates which reflect the semantics of the partition. The following examples specify partition classes for Person, inherently constituting a complete and disjoint partition of Person.

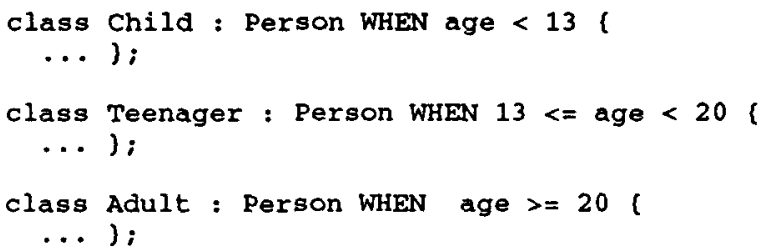

Adding the following means that the partition is (potentially) no longer disjoint:

class Retired : Person WHEN age $>=67$ (

... \};

Adding the following implies there will be multiple partitions of Person:

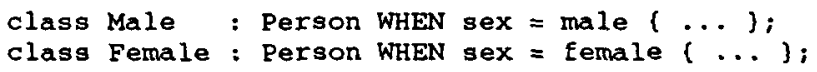

Automatic category classes may also be applied to define various propagations of class membership, on the basis of different criteria. These category classes are typically defined by role-based predicates, and so that possession of a special combination of roles implies automatic assumption of another role. Consider the following example:

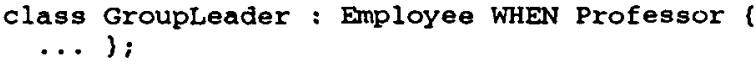

As a general rule, every full professor is entitied to her own group, in which the professor automatically becomes the group leader. However, the class GroupLeader is not a specialization of Professor: The fact that all group leaders are professors (and vice versa) is just another role that inherently is played by professors. Groupleader and Professor are sibling classes in the class hierarchy; both are subclasses of Employee.

More complex propagation specifications may be defined using $\mathrm{C}++$ logical operators, e.g. to define new classes as intersections, unions or differences between others. Due to the notion of object roles there is no special restrictions wrt. the placement of such classes in hieranchy ${ }^{8}$. For instance, a temporary employee is any employee which is not a faculty member or a technicalladministrative person:

${ }^{8} \mathrm{COCOON}$ [SS91], for instance, requires that an intersection class is a subclass and a union class a superclass of the base classes. 
Property and role-based predicates may be combined, and so that membership in the category class is based on both property evaluation and role possession. This may be convenient to model conditional propagation. For instance, a researcher is automatically promoted to a senior researcher after ten years of employment, provided she has a $P h D$ degree:

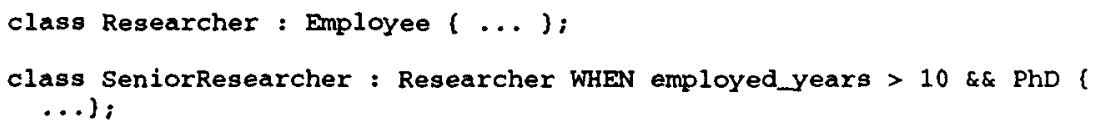

Two-way propagation is useful when two classes, which are sibling classes, should contain the same objects $^{9}$. For instance, for the employees at some university, all lecturers are faculty members, and vice versa:

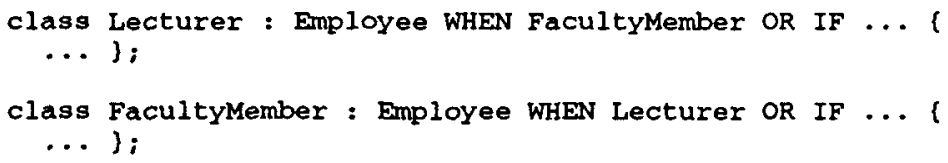

Lecturer and FacultyMember are sibling classes in the class hierarchy, so that any person possessing either of these roles will also (automatically) possess the other (and generally behave in different ways in the different roles). The definition of classes Lecturer and FacultyMember also includes an IF expression, ensuring that roles corresponding to these classes may be explicitly added as well; otherwise there will be no way for any object to become a lecturer or faculty member. The combination of manual and automatic predicate expressions is elaborated upon in the next section.

\subsection{Manual and automatic category classes}

Category classes which are both manual and automatic may have membership criteria defined by a conjunction (AND) or disjunction (OR) between IF and WHEN expressions (associated with the same or different candidate classes $\left.{ }^{10}\right)$ :

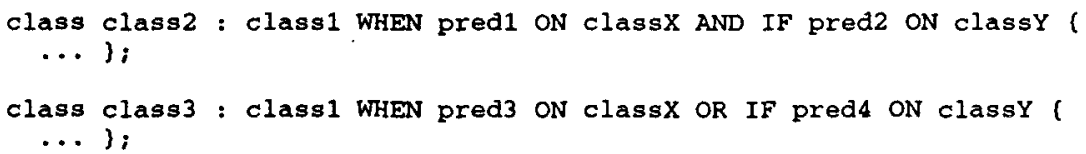

class 2 is defined by conjunction, and the corresponding role may be assumed by an object upon explicit addition only, and only in the case that both predicates pred1 and pred2 (defined on the candidate class class $X$ and class $Y$, respectively) are satisfied. The role will be removed from the object by explicit request (using the rem operator, and irrespective of predicate evaluation), or implicitly if pred1 is no longer satisfied. In the latter case the role may reappear upon possible re-satisfaction of the predicate.

Conjunction-based category classes are most interesting in cases where the role must be explicitly added, although may also be automatically lost. For instance, only persons which are older than eighteen may become an employee (assuming this is the law for some particular work). When an employee retires, the employee role is automatically lost.

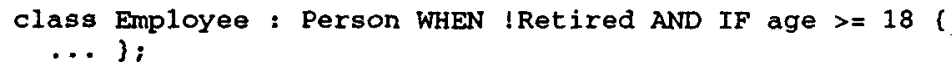

\footnotetext{
'If one class is a subclass of the other, propagation into the superclass will be implicit due to substitutability.
}

${ }^{10}$ Most frequently they associate with the same. 
This is one example on the modeling of a transition for which the assumption of one role necessarily implies that some other (which does not reflect a superclass) must be lost. Another examples models how an associate professor role is revoked when promoted to a full professor (Professor is not a subclass of AssocProfessor):

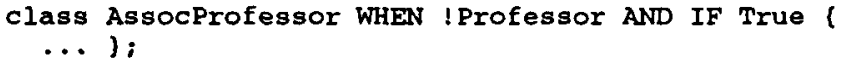

class 3 is defined by disjunction, and the corresponding role is added to some object either implicitly by the satisfaction of predicate pred3 or upon explicit request (provided that predicate pred4 is satisfied). Removal of the role from the object is dependent on the way it was added. If the role has been implicitly added (through the satisfaction of prod3) and pred3 is no longer satisfied, the role is removed from the object. If role removal is explicitly requested, the role is removed uniess pred3 is satisfied. Note that it is impossible to remove explicitly a role which have been implicitly added. Furthermore, implicitly removed roles may reappear upon some future re-satisfaction of the pred3 predicate.

An example of a disjunction-based manual and automatic category class is the class Retired used above. In Norway, a person generally retires at 67 , but may continue working until 70 when she must retire ${ }^{11}$ :

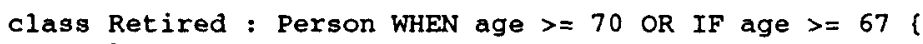

\subsection{Disjoint Predicates}

In many cases we find that there are natural restrictions on role possession, and which are, using category classes, directly associated with each class. However, frequently we find that the restriction is more naturally associated with a complete collection of classes, and so that possession of a role corresponding to either of these inhibits the possession of others from the same collection. For instance, possession of roles corresponding to classes Child, Employee, Retired and Dead is inherently disjoint, and may be specified as follows ${ }^{12}$ :

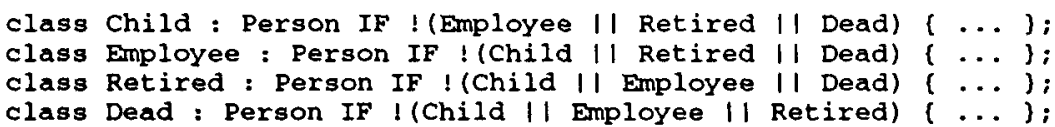

As a simplified specification of this relationship between classes, the notion of a disjoint predicate is introduced. Disjoint predicates specify that no object may be a member of more than one class in a specified collection at the same time. If an object already is an instance of one of the indicated classes (or a subclass of this class), it is not possible to add (explicitly or implicitly) any of the other classes, unless the former class is eventually removed from the object. Using disjoint predicates, the definitions above may be rewritten as:

disjoint (Child, Employee, Retired, Dead) :

Disjoint predicates may range over both manual and automatic category classes, but most frequently apply for manual category classes as they define inherent restrictions on object evolution and role combination. Automatic category classes are enabling in nature, and thus definitions easily may conflict with disjoint predicates ${ }^{13}$. Furthermore, disjoint predicates are solely role based, and thus cannot reference properties of classes participating in the predicate. Disjoint predicates represent a convenient shorthand specification mechanism, however may always be rewritten in terms of equivalent restrictions directly associated with the classes involved.

\footnotetext{
${ }^{11}$ In reality, rules for retirement are more complex.

${ }^{12}$ Other restrictions may also be associated with these category classes, but are left out here for brevity.

${ }^{13}$ The class Child above may, however, typically be an automatic category class.
} 


\section{Comparison}

The notion of an object role is not new, and various appròaches to providing more support for multi-perspectived objects and flexible object evolution have been described $i$ literature. In most traditional object models a multi-perspectived nature of objects may only be described through multiple inheritance, defining special "intersection classes". However, these classes are constructed abstractions which need not reflect any natural abstraction from the real world. Furthermore, a single behavioral context is imposed, when multiple independent contexts are more natural, and there is a possibility for a combinatorial explosion of intersection classes to model all possible combinations. McAllester [MZ86] introduce a notion of boolean classes to alleviate this problem. Clovers [SZ89] is particularly concemed about the ability for objects to possess multiple independent perspectives, how these may be added, and how they are independently referenced. However, while new roles (or leaves in the "clover") may be added, they cannot be removed. Consequently, Clovers may hardly be regarded to properly support evolving objects. OORASS [RAB +92 ] is more concerned about the role as the fundamental concept, describing patterns of communication between roles and how instances of different types may play one role.

Other approaches are more concemed about the flexible classification of objects through predicate (over object state) satisfaction. In this way objects may implicitly evolve, on the basis of intensional descriptions. Many approaches to OODB views have been proposed, for which objects may assume membership in virtual classes based on the satisfaction of a selection predicate. However, virtual (view) classes often cannot be managed in the same way as ordinary classes. COCOON [SS91] is one example, and will be described below. ECR [EWH85] provides flexible capabilities to define how instances of a class may be specialized into subclasses, and how these may define disjoint/nondisjoint and complete/non-complete partitions of a superclass. [Cha93] presents a notion of predicate classes, which are similar to ordinary classes, but membership may only be assumed automatically by predicate satisfaction. Special restrictions on class membership combination may be imposed ${ }^{14}$. Predicate classes are similar to our automatic category classes, however are mostly concemed about the possibility for state-specific object specializations for dispatching purposes (and assumption of additional attributes).

Some approaches are concemed about object evolution on explicit (extensional) request. Aspects [RS91] allows for arbitrary addition and removal of special "aspects" (chunks of state and behavior) to existing objects. Multiple aspects may be added to objects, which thus may behave in a multiperspectived manner. The aspect definition is regarded as an extension to some ordinary class, but will not inherit strictly from this class. This means that aspects do not integrate properly with the ordinary class hierarchy, with the possible implication that an employee aspect addition to a person may no longer be acceptable as a person. Iris $\left[\mathrm{FBC}^{+} 87\right]$ also allows for arbitrary addition and removal of types dynamically, however there is only one context of behavior, and thus no notion of role. [Zdo90] allows for roles to be added and removed, providing special abilities to specify that some roles are not removable ("essential type"), and that some roles may only be acquired upon creation ("exclusionary type"). Based on these, other restrictions on evolution may be specified, although in an awkward and unnatural way. [Ara89] defines conversions (change class) and enhancements (add class) to objects through special functions associated with the source class of the migration, and which define all valid migration patterns of objects.

Finally, [Ve193, SS91] provide abilities for both implicit and explicit evolution (classification) of objects. [Vel93] allows object to be specialized/generalized (to a subclass/superclass) upon the occurrence of an event (a method invocation), provided that an associated assertion is satisfied. As both these are optional, a transition may be completely implicit (no event), or explicit (no assertion). Life cycles (sequences of valid event occurrences) may be defined to restrict valid patterns of evolution. To model the fact that instances of different classes may play the same role, a transition to one class may originate from different source classes. However, in this way the destination class (denoted a phase) will behave differently from ordinary classes wrt. inheritance and

\footnotetext{
${ }^{14}$ These restrictions are motivated by the need to ensure that some objects may not be a member of multiple, most-specific classes when a binding conflict may occur.
} 
substitutability. That is, the notion of a phase is a (partially) different abstraction mechanism from the class. COCOON [SS91, SLT91] describes an OODB view mechanism which distinguishes type (interface) and class (collection of objects with the same type). These are organized in different (but often correlating) hierarchies for property inheritance and subsetting, respectively. Classes may be associated with a predicate (over the state), stating necessary (corresponding to our manual) or necessary and sufficient (corresponding to our automatic, and reflecting a select query) conditions on membership. In this way objects may dynamically evolve (by gaining/losing class membership) implicitly or upon explicit request, which may also imply the assumption of more type. However, these types are virtual (i.e. derived from other types), and thus objects cannot assume new state. COCOON also provides special set-theoretic operators for the definition of view classes, with special rules for how these are to be placed in the class hierarchy). No arbitrary propagation of membership into sibling classes are, however, possible. Furthermore, no combination of manual and automatic specifications (which was found to be very useful in Section 3.4) may be given. COCOON is primarily a view approach, and thus more concemed about how virtual classes and types are to be located in the class/type hieranchies, and how they map onto base classes/types.

\section{Conclusions, Contributions and Further Work}

A notion of an object role, describing a perspective of an object as an instance of a particular class, has been presented. Roles may dynamically be added or removed from objects, according to how real-world objects evolve and exhibit themselves through multiple perspectives. Category classes describe constraints on valid evolution pattems and combination of roles, as well as defining how the possession of a role may be automatically enabled. Finally, category classes constitute a powerful vehicle for conceptual modeling, with flexible means for object classification. While the model has been presented in a database context, the important aspects have more general applicability as a powerful modeling framework or part of a programming language.

The major contributions of the approach relate to the way flexible classification, object evolution and the multi-perspectived nature of objects are smoothly integrated within an object model based on C++. This is achieved by retaining the same abstraction mechanism (the class) as the basis for classification and addition/removal of roles. In this way the importance of the class hierarchy as an organization of real-world knowledge (conceptual specialization and property inheritance) is not affected by the added modeling power. The approach integrates multiple classification mechanisms: Class-based classification is defined by ordinary classes, and set-based classification may be defined extensionally (by manual category classes) and intensionally (by automatic category classes). Membership in different classes may be regarded independently, as different perspectives of the same object. A particularly useful provision is the ability to propagate objects (possibly conditionally) into other classes, i.e. acquiring new roles on the basis of some particular (combination of) role(s) possessed. The approach allows objects to evalve over time with special facilities to restrict and enable object evolution based on both the state and mole possession of the object. In this way objects may explicitly and implicitly gain and lose roles and properties dynamically, without compromising the class hierarchy as the conceptual organization of real-world knowledge.

A prototype OODB, incorporating the notions of object role and category classes, is being implemented to demonstrate the applicability of the ideas. Preliminary results are promising. However, the primary focus of our work is within the area of schema versioning, and how different schema versions may contain different versions of the same class. [Odb94] will show how the evolutionary and multi-perspectived nature of objects have many traits which are similar to the evolutionary and multi-perspectived nature of classes, and how many principles of management are the same.

Acknowledgments Svein Erik Bratsberg and Reidar Conradi are acknowledged for comments and discussions. 


\section{References}

[Ara89] Constantin Arapis. Type Conversion and Enhancement in Object-Oriented Systems. In D. Tsichritzis: Object Oriented Development, pages 191-205. Centre Universitaire d'Informatique, Université de Genève, July 1989.

[Cha93] Craig Chambers. Predicate Classes. In ECOOP '93. European Conference on ObjectOriented Programming, Kaiserslautern, Germany, July 1993.

[EWH85] R. Elmasri, J. Weeldreyer, and A. Hevner. The Category Concept: An Extension to the Entity-Relationship Model. International Journal of Data \& Knowledge Engineering, 1, May 1985.

[FBC ${ }^{+87]}$ D.H. Fishman, D. Beech, H.P. Cate, E.C. Chow, T. Conners, J.W. Davis, N. Derrett, C.G. Hoch, W. Kent, P. Lyngbaek, B. Mahbod, M.A. Neimat, T.A. Ryan, and M.C. Shan. Iris: An Object-oriented Database Management System. ACM Transactions on Database Systems, January 1987. Also in [ZM90].

[MZ86] David McAllester and Ramin Zabih. Boolean Classes. In Proceedings of the Conference on Object-Oriented Systems, Languages and Applications(OOPSLA), Portland, Oregon, USA, pages 417-423, September 1986.

[Odb92] Erik Odberg. What "What" is and isn't: On Query Languages for Object-Oriented Databases. Or. Closing the Gap - Again. In TOOLS USA ' 92 (Technology of ObjectOriented Languages and Systems), Santa Barbara, California, USA, August 1992.

[Odb94] Erik Odberg. MultiPerspectives: Object Evolution and Schema Modification Management in Object-Oriented Databases. PhD thesis, Department of Computer Science, Norwegian Institute of Technology, 1994. In preparation.

[RAB +92] Trygve Reenskaug, Egil P. Andersen, Ame Jørgen Berre, Anne Hurlen, Anton Landmark, Odd Arild Lehne, Else Nordhagen, Eirik Nass-Ulseth, Gro Oftedal, Anne Lise Skaar, and Pal Stenslet. OORASS: Seamless Support for the Creation and Maintenance of ObjectOriented Systems. Journal of Object-Oriented Programming, 5(6):27-41, October 1992.

[RS91] Joel Richardson and Peter Schwarz. Aspects: Extending Objects to Support Multiple, Independent Roles. In Proceedings of ACM/SIGMOD (Management of Data), Denver, Colorado, pages 298-307, 1991.

[SLT91] Marc H. Scholl, Christian Laasch, and Markus Tresch. Updatable Views in ObjectOriented Databases. In Proceedings of the Second International Conference on Deductive and Object-Oriented Databases (DOOD91), Munich, Germany, December 16-18, 1991, pages 189-207, December 1991.

[SS91] Marc H. Scholl and H.-J. Schek. Supporting Views in Object-Oriented Databases. IEEE Data Engineering Bulletin, 14(2), June 1991.

[SZ89] Lynn Andrea Stein and Stan Zdonik. Clovers: The Dynamic Behavior of Types and Instances. Technical report, Brown University, Department of Computer Science, November 1989. Technical Report No. CS-89-42.

[Ve193] Amandio de Jesus C. Vaz Velho. From Entity-Relationship Models to Role-Attribute Models. In Proceedings of the 12 th International Conference on the Entity-Relationship Approach, Arlington, Texas, December 1993.

[Zdo90] Stanley B. Zdonik. Object-Oriented Type Evolution. In François Bancilhon and Peter Buneman (Eds.): Advances in Database Programming Languages, chapter 16, pages 277-288. Addison-Wesley, 1990.

[ZM90] Stanley B. Zdonik and David Maier, editors. Readings in Object-Oriented Database Systems. The Morgan Kaufman series in Data Management Systems. Morgan Kaufman, 1990. ISBN 0-55860-000-0. ISSN 1046-1698. 